Accepted Version. This article is published as: H.W. Wopereis, L.W. van de Ridder, T.J.W. Lankhorst, L. Klooster, E.M. Bukai, D. Wuthier, G. Nikolakopoulos, S. Stramigioli, J.B.C. Engelen and M. Fumagalli,

"Multimodal Aerial Locomotion: An Approach to Active Tool Handling", in IEEE Robotics and Automation Magazine, vol. 25, no. 4, pp. 57-65, Dec. 2018. Doi: 10.1109/MRA.2018.2869527 @ 2018 IEEE

\title{
Multimodal Aerial Locomotion: An Approach to Active Tool Handling
}

\author{
H.W. Wopereis ${ }^{1}$, L.W. van de Ridder ${ }^{1}$, T.J.W. Lankhorst ${ }^{1}$, L. Klooster ${ }^{1}$, E.M. Bukai ${ }^{1}$, D. Wuthier ${ }^{3}$, \\ G. Nikolakopoulos ${ }^{3}$, S. Stramigioli ${ }^{1}$, J.B.C. Engelen ${ }^{1}$ and M. Fumagalli ${ }^{2}$
}

\begin{abstract}
This work reports on the development and evaluation of an aerial system for active tool handling on remote locations. In the proposed approach a multirotor UAV is responsible for moving an end-effector with a tool to the region of interest and providing sufficient contact force for the endeffector to accomplish the desired task. The end-effector is equipped with actuated wheels that rely on the contact force to both allow an operator to re-position while in contact with the environment and perform the tool operation.

Preliminary experiments validate the approach in a cleaning scenario and demonstrate the repeatability in an experiment with 18 consecutive repetitions of the approach.
\end{abstract}

\section{INTRODUCTION}

The focus of research in aerial robotics started shifting from contactless inspection towards interaction and manipulation, with the number of potential applications rapidly expanding [1]. In the long term, aerial manipulators, i.e. UAVs equipped with manipulators, are expected to relieve humans from maintenance routines under hazardous conditions. For this to happen, aerial manipulators need to become able to perform all the different operations that are required in these maintenance routines.

Many of these operations require the aerial manipulator to either move or position the tool precisely on the surface of a static object, while generating sufficient contact force on the tool for correct operation. Examples of such operations are cleaning, grinding or contact-measurements. Two main challenges are faced simultaneously in these operations: being able to exert (persistent) large contact forces to the tool, that are necessary to perform meaningful operations on the surface, and being able to move the tool over the surface on which the operation takes place. These challenges are even more problematic when the aerial platform is underactuated, as is the vast majority of commercially available aerial platforms. For this class of systems the dynamics are altered in interaction due to contact constraints, which introduces additional coupling terms between the linear and rotational dynamics [2]. This, in combination with the anisotropy of the torque-limits, complicates the task of both applying relevant contact force and moving the end-effector on the surface.

* This work has been funded by the European Commission's H2020 project AEROWORKS under grant no. 644128.

${ }^{1}$ Faculty of Electrical Engineering, Mathematics and Computer Science, CTIT Institute, University of Twente, The Netherlands. Email: $\{$ h.w.wopereis, j.b.c.engelen, s.stramigioli $\} @$ utwente.nl

${ }^{2}$ Robotics, Vision and Machine Intelligence (RVMI) Lab, Dept of Mechanical and Manufacturing Engineering, Aalborg University, Copenhagen, Denmark. Email: m_fumagalli@m-tech.aau.dk

${ }^{3}$ Department of Computer, Electrical and Space Engineering, Luleå University of Technology, Luleå, Sweden. Email: geonik@ltu.se

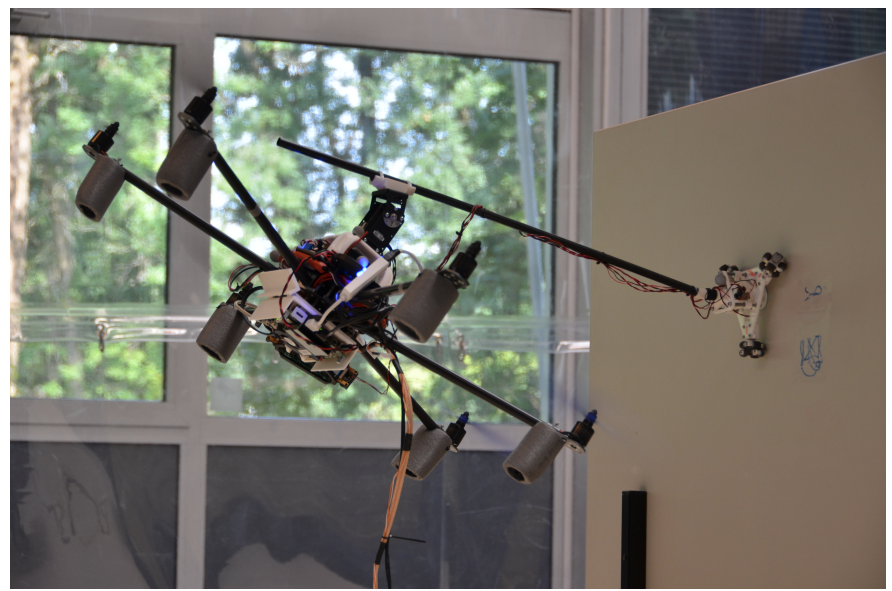

Fig. 1: Photo demonstrating the multimodal locomotion system performing a cleaning operation on a vertical surface.

Regular multirotors-multirotors with coplanar rotorstypically use classical control approaches to maintain stability up to certain interaction forces [3], where the boundaries for stability are assessed in [4]. This approach has been adopted in [5] to apply a quadrotor as a 3D force effector. Typically, in scenarios that require substantial interaction forces, wide placed contacts are used to constrain the rotational dynamics, while additional [6] or tilting rotors [7], or the full body rotation [8] is used to generate the contact force. However, the use of wide contacts restricts the systems motion capabilities.

Instead of regular multirotors, fully-actuated multirotors can be used as the floating base platform for the aerial manipulator, as these do not suffer from the intrinsic challenges posed by underactuation [9]. In this case, rotors can be placed in different non-coplanar configurations [9], [10] or mechanisms can be designed that adjust the orientation of the rotors with respect to the body-fixed frame [11]. However, these systems have the drawbacks that additional mechanisms introduce weight to the system and that, in noncoplanar configurations, some rotors may not fully contribute to the generated output thrust.

Only few results have been presented in which active locomotion of a tool over a surface is achieved, where a UAV is used as the driving factor for the locomotion of the tool. Such UAV-driven approaches have demonstrated capable of active movement over a surface [12], [13], while only applying limited interaction forces. 


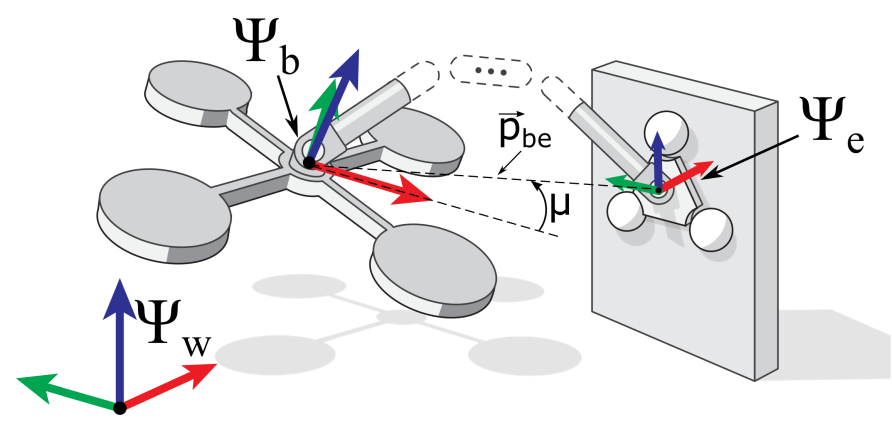

Fig. 2: Illustration of the aerial manipulator showing the inertial (world) frame $\Psi_{w}$, the body-fixed frame of the multirotor $\Psi_{b}$ and the end-effector frame $\Psi_{e}$. The red, green and blue axes represent $(\hat{x}, \hat{y}, \hat{z})$, respectively.

In this work, as an alternative to UAV-driven locomotion, we introduce the concept of multimodal aerial locomotion for active tool handling in aerial manipulation. Multimodal locomotion demonstrated to provide additional locomotion capabilities in environments where a single modality would not allow the accomplishment of the task [14], [15]. We present a novel platform that exploits two locomotion modalities in order to achieve precise tool positioning and persistent contact force. Precisely, we combine an aerial manipulator with a wheeled end-effector. Three actuated omnidirectional wheels are placed on this end-effector to allow active locomotion of the tool, which require sufficient friction with the surface to function correctly. To guarantee this friction, a controller based on our previous work [2] is applied. The scope of this controller is twofold: it provides the contact force that is necessary to ensure wheeled locomotion and tool operation, and it stabilizes the attitude of the UAV subject to the interaction forces. The end-effector is presented in Section III], which represents the first contribution of this paper. The controller is presented in Section IV] which, together with its use for multimodal aerial locomotion, represents the second contribution of this paper. The approach has been evaluated in experiments in which the end-effector, mounting a cleaning brush, is used to clean a marker drawing on a flat vertical surface. The results of these experiments demonstrate effective locomotion of both the aerial and wheeled system, thus validating our approach to multimodal locomotion for active tool handling.

\section{Multimodal Aerial Locomotion Approach}

The main task envisioned in this work is the cleaning of a confined region on a static surface with a soft brush, resembling a maintenance operation on a wind-turbine. In order to demonstrate the multimodal locomotion approach we consider a challenging scenario: moving an end-effector on a vertical surface. For sake of simplicity but without loss of generality regarding the approach, we consider a flat rigid surface and we assume the position and orientation of the surface at the region of interest to be known.

Within this scenario, our multimodal locomotion approach to perform the required surface operation task uses an aerial manipulator with an end-effector that mounts the brushing tool and wheels that allow for active re-positioning on the surface. This is sketched in Figure 2.

The multimodal locomotion system consists of a regular multirotor, a 1-DoF manipulator and a custom designed endeffector. The multirotor constitutes the aerial-locomotionsystem 1 and functions as the base for the manipulator. On one extremity, the manipulator contains a 1-DoF joint, which connects to the multirotor and decouples the pitch of the multirotor. On the other extremity, a flexible joint connects the manipulator and the end-effector, decoupling their relative orientation. The end-effector is used to perform the ground locomotion 2 by means of an omnidirectional driving unit, which enables re-positioning of the tool and its operation.

The symbiosis between the aerial-locomotion-system and ground-locomotion-system is the key-element that allows the task to be accomplished. The aerial-locomotion-system carries the tool to the desired location, while the accuracy in positioning the tool is achieved by the ground-locomotionsystem. A normal force between the surface and the wheels is required to generate friction for the ground-locomotionsystem to work. In the scenario of cleaning a vertical surface, this normal force can only be achieved by the aerial system pushing the ground-locomotion-system onto the surface.

As a consequence of coupling the aerial-locomotionsystem to the ground-locomotion-system, we need to guarantee that displacements of the ground-locomotion-system do not affect the stability of the aerial-locomotion-system. Due to the presence of constraints, movements of the groundlocomotion-system affect the dynamic behavior of the aerial system. This introduces disturbances in the form of forces applied to the tip of the manipulator, which results in a rotation and displacement of the aerial system. To deal with this undesired effect, we propose to use a modified controller that is based on our previous work [2]. This contact controller stabilizes the relative orientation of the multirotor to the end-effector from disturbances caused by the coupling with the end-effector, which results in automatic tracking of the ground locomotion. In practice, this implies that any perturbation introduced to the multirotor by the motion of the end-effector results in a new equilibrium position to which the multirotor will stabilize. This self-stabilization allows us to control the position of the multirotor implicitly by controlling the position of the ground-locomotion-system. An elastic element provides the rotational decoupling necessary to facilitate this effect. The rotational compliance introduces a spherical constraint which allows relative displacements between the multirotor and the end-effector. This is needed to allow the contact controller to react to the displacement within its bandwidth. Besides stabilizing the system, the

\footnotetext{
${ }^{1}$ We define aerial locomotion, the locomotion occurring in an unconstrained environment. An aerial-locomotion-system is therefore defined as a system that is unconstrained to a static environment.

${ }^{2}$ We define ground locomotion as the locomotion on a static environment and a ground-locomotion-system as a system that performs locomotion on a static environment.
} 


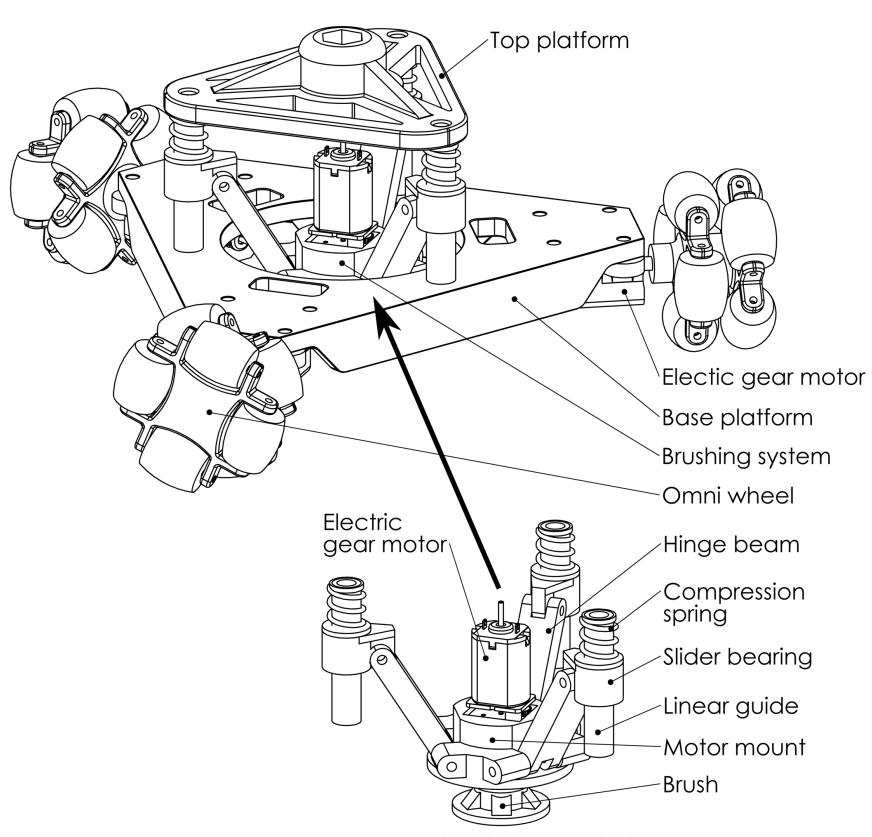

Fig. 3: Illustration of the end-effector design.

contact controller has the task of providing the normal forces required for successful ground locomotion and tool operation.

\section{END-EFFECTOR DESIGN}

The end-effector, which is detailed in Figure 3 , is designed with the functionality to move along the surface and perform surface operations (in this case brushing). Both these functions rely on the presence of normal force between the end-effector and the surface. Therefore, in the mechanical design of the end-effector emphasis is put to ensure a robust yet lightweight system.

The body of the end-effector is formed by the baseplatform and top-platform, which are rigidly connected by three metal spacers. To add the functionality of moving along the surface, three actuated omni wheels are attached to the base-platform at $120^{\circ}$ angles. Each of these wheels is actuated in one direction and contains freely rotating barrels that allow movement in the other direction. This combination of three independently driven wheels results in full controllability of the end-effector pose on the surface, assuming sufficient friction.

The brushing system, as detailed in the bottom of Figure 3 . represents the surface operation functionality. Different types and sizes of tools can be used, which may require different surface pressure depending on the operation. To control this surface pressure, the tool is mounted on a parallel structure which is suspended by three compression springs. The parallel structure is composed of three hinge beams that connect the motor mount to linear slider bearings, which slide over the linear guides. These linear guides are in fact the spacers between the top and bottom platform. The material stiffness of the beams allows minor rotational misalignment of the tool. In the uncompressed state, the

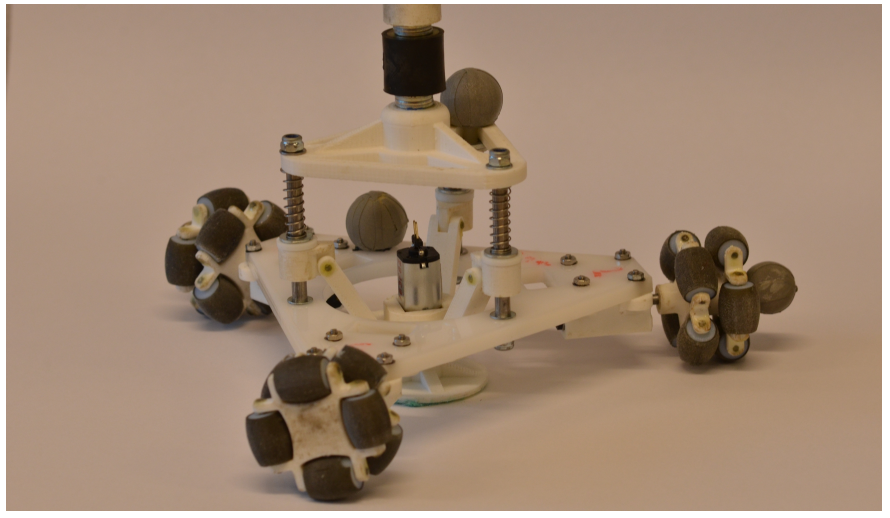

Fig. 4: Photo of the end-effector. Except for the metal components, motor and flexible element, all parts have been produced using rapid-prototyping techniques.

springs press the brushing system against the base platform so that the brush sticks out. When compressed, with all wheels being in contact with the surface, the springs apply a constant force on the brushing system, independent of the contact force of the drone.

The prototype of the end-effector (Figure 4) weighs $0.15 \mathrm{~kg}$ and carries a flat soft brush with a diameter of $3 \mathrm{~cm}$. The suspension is designed to apply a force of $6 \mathrm{~N}$ in the compressed state, which translates in an applied pressure of $8500 \mathrm{~Pa}$. Four high-power 300:1 Pololu Micro Metal Gearmotors are used for actuating the wheels and the brush, which are controlled by an Adafruit Feather board. The ground locomotion is controlled by an open-loop controller with Cartesian body-velocity commands ranging from $[-1,1]$ as input. The flexible joint connecting the endeffector and the manipulator is implemented as a a male-tomale M5 rubber shock mount with a compression load of 200 N, shown at the top in Figure 4

\section{Control Strategy}

We define the frames in our system as illustrated in Figure 2. Frames $\Psi_{\mathrm{w}}, \Psi_{\mathrm{b}}$ and $\Psi_{\mathrm{e}}$ represent the inertial (world) frame, the body-fixed frame of the multirotor and the end-effector frame, respectively. Frame $\Psi_{\mathrm{b}}$ has its origin in the center of mass of the multirotor, with $\hat{x}_{\mathrm{b}}$ aligned with the forward direction and $\hat{z}_{\mathrm{b}}$ with the thrust vector. Frame $\Psi_{\mathrm{e}}$ is oriented as $\Psi_{\mathrm{w}}$ and has its origin in the elastic component connecting the end-effector to the manipulator. The roll, pitch and yaw angles of the multirotor about $\left(\hat{x}_{\mathrm{b}}, \hat{y}_{\mathrm{b}}, \hat{z}_{\mathrm{b}}\right)$ are denoted by $\left(\phi_{\mathrm{b}}, \theta_{\mathrm{b}}, \psi_{\mathrm{b}}\right)$, respectively. We assume that the origin of frame $\Psi_{\mathrm{e}}, p_{\mathrm{e}}$, always lies in the $\left(\hat{x}_{\mathrm{b}}, \hat{z}_{\mathrm{b}}\right)$ plane. This implies that the pitch angle and thrust of the multirotor define the magnitude of the contact force. The manipulator angle $\mu$ is given by the angle between the axis $\hat{x}_{\mathrm{b}}$ and the vector $\vec{p}_{\mathrm{be}}$ expressed in $\Psi_{\mathrm{b}}$, and positive rotation is defined counterclockwise with respect to $\hat{y}_{\mathrm{b}}$. We assume a constant distance between $\Psi_{\mathrm{b}}$ and $\Psi_{\mathrm{e}}$, annotated by $L_{\mathrm{m}}$.

Considering our application scenario, four phases for the system can be identified: free-flight, engage, contact and 
disengage. The engage phase is the period in which the approach is initiated until the system is in stable contact. The disengage phase occurs from the moment the disengage is initiated until the system has recovered to its position setpoint, placed at a fixed distance $C$ from the surface. At the start of the engage phase we require that the end-effector is in proximity of the surface $(<30 \mathrm{~cm})$.

The switching control strategy used during the different phases is illustrated in Figure 5. Two separate controllers are used: a free-flight controller and a contact controller. Depending on the phase, different inputs are given to these controllers and different controller outputs are used. The same applies for the manipulator setpoint. Both controllers receive the state information of the multirotor: position $p_{\mathrm{b}}$, velocity $v_{\mathrm{b}}$, orientation $R_{\mathrm{b}}^{\mathrm{w}}$ and angular rates $\omega_{\mathrm{b}}$.

As can be observed in Figure 5, the free-flight controller is used in free-flight phase with the manual setpoints and during disengage with a constant setpoint $C$ from the surface, determined at the start of disengage. In the engage phase, the implementation of the contact controller as presented in [2] is used, with as reference inputs the three desired body angles and height setpoint, given by $z_{s p}=z_{\mathrm{b}}\left(t_{\mathrm{e}}\right)$, with $t_{\mathrm{e}}$ being the time engage mode was entered. During contact, the modified implementation of the contact controller, as described in Section IV-A.2, is used so that the controller solely depends on angular setpoints and angular state measurements. This controller is position-independent, which allows the multirotor to track the ground-locomotion without active coordination between the setpoints of the endeffector and the multirotor.

Since a vertical surface is assumed, in free-flight, engage and disengage, the manipulator is given the setpoint $\mu_{\mathrm{sp}}=$ $\theta_{\mathrm{b}}$, so that the end-effector is always in front of $\Psi_{\mathrm{b}}$. The manipulator is assumed sufficiently fast to accurately track $\theta_{\mathrm{b}}$. In contact the manipulator setpoint is set to a specified value $\mu_{\mathrm{sp}}=\mu_{\mathrm{c}}$, which follows from the conditions required for equilibrium.

\section{A. Control algorithms}

1) Free-flight controller: The free-flight controller in this work consists of a generic cascaded position controller. It consists of four different stages, where each subsequent stage takes the output of the previous stage as the reference input. In order, these controllers are: a proportional $(\mathrm{P})$ controller on the position, the velocity and the attitude errors, and a PID controller on the angular rate error. Due to the cascaded structure, subsequent control stages act as damping on the previous stages. Additional integral and derivative actions are added to the rate-control loop to increase the tracking performance of the angular rates. Furthermore, gravitycompensation is taken into account and a static center of mass correction is applied. The latter is required because the additional weight of the manipulator and end-effector affect the center of mass of the total system. Given the structure of the manipulator used in this work, the variations in the center of mass are considered negligible. As such, the center of mass correction yields a constant compensation torque

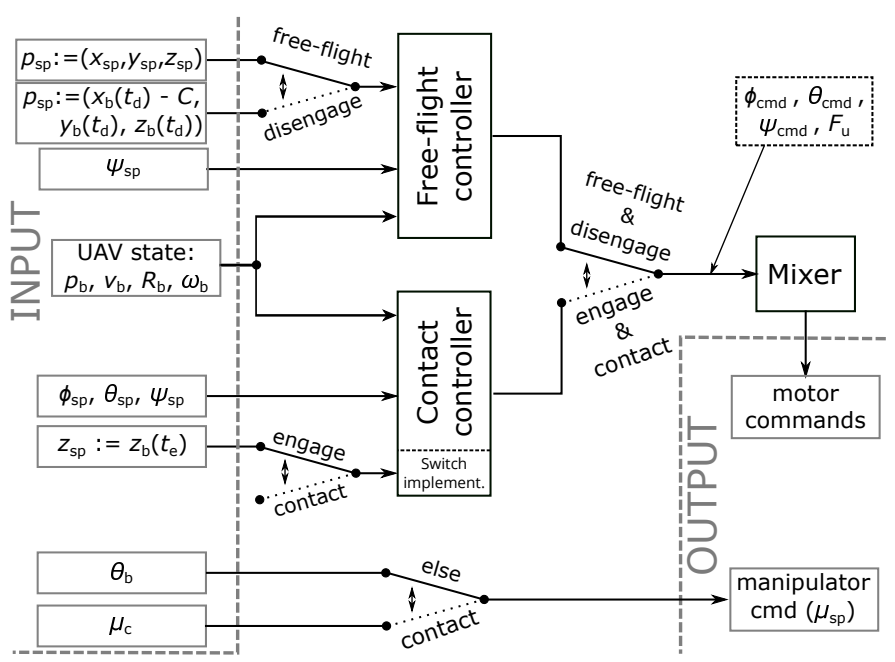

Fig. 5: Schematic illustrating the switching control strategy. Depending upon the phase, different inputs and outputs are selected.

about $\hat{y}_{\mathrm{b}}$. The value is found empirically by readjusting the compensation torque until the setpoint and actual position in free-flight match.

2) Contact controller: The contact controller is based on the work presented in [2] and is specifically designed for when the system is in contact with the environment. We demonstrated that stability in contact can be maintained while simultaneously applying a substantial contact force. This result was achieved by actively exploiting the coupling between the roll and yaw state in contact, while retaining the regular controllers for the pitch and altitude. As a contribution of this work, a modified version of the controller presented in [2] is derived, specifically for the contact phase, which relies solely on angular state measurements to maintain its orientation relative to the end-effector and is therefore fully independent of any position measurements. This allows the multirotor to automatically track movements of the end-effector without the need of active adjustments to its setpoints.

In this derivation we consider the end-effector to be fixed to the surface due to the friction and normal force, and the manipulator to be connected to the end-effector by a spherical joint in $\Psi_{\mathrm{e}}$. Furthermore, we assume that the static vertical surface is oriented so that $\hat{x}_{\mathrm{e}}$ is perpendicular to it and points inwards. Applying screw theory [16] and ignoring frictional effects, the equations of motion expressed in $\Psi_{\mathrm{b}}$ can be described as:

$$
I_{\mathrm{b}} \dot{T}_{\mathrm{b}}^{\mathrm{b}, \mathrm{e}}=\mathrm{ad}_{T_{\mathrm{b}}^{\mathrm{b}, \mathrm{e}}}^{\mathrm{T}} I_{\mathrm{b}} T_{\mathrm{b}}^{\mathrm{b}, \mathrm{e}}+\operatorname{Ad}_{H_{\mathrm{b}}^{\mathrm{g}}}^{\mathrm{T}}\left(W_{\mathrm{g}}\right)^{\mathrm{T}}+\left(W_{\mathrm{b}}\right)^{\mathrm{T}}+\operatorname{Ad}_{H_{\mathrm{b}}^{\mathrm{e}}}^{\mathrm{T}}\left(W_{\mathrm{e}}\right)^{\mathrm{T}} .
$$

Here, $I_{\mathrm{b}} \dot{T}_{\mathrm{b}}^{\mathrm{b}, \mathrm{e}}$ is the change in momentum of the multirotor with respect to $\Psi_{\mathrm{e}}$, with $I_{\mathrm{b}}$ the inertia of the multirotor and $T_{\mathrm{b}}^{\mathrm{b}, \mathrm{e}}$ the relative twist of $\Psi_{\mathrm{b}}$ to $\Psi_{\mathrm{e}}$ expressed in $\Psi_{\mathrm{b}}$. The fictitious forces are accounted for by $\operatorname{ad}_{T^{\mathrm{b}, e}}^{\mathrm{T}} I_{\mathrm{b}} T_{\mathrm{b}}^{\mathrm{b}, \mathrm{e}}$ ( $\Psi_{b}$ is not an inertial frame). The wrenches $W_{\mathrm{g}}, W_{\mathrm{b}}$ and $W_{\mathrm{e}}$ are the gravity, input and contact wrench, respectively. $H_{\mathrm{i}}^{\mathrm{j}} \in \mathrm{SE}(3)$ 
is the homogeneous transformation matrix from $\Psi_{\mathrm{i}}$ to $\Psi_{\mathrm{j}}$. The matrix $\operatorname{Ad}_{H_{\mathrm{i}}^{\mathrm{j}}}^{\mathrm{T}}$ describes the transformation of a given wrench from frame $\mathrm{j}$ to frame $\mathrm{i} . \Psi_{\mathrm{g}}$ is the gravitational frame, which coincides with $\Psi_{\mathrm{b}}$ and is oriented as $\Psi_{\mathrm{w}}$. Note, $H_{\mathrm{b}}^{\mathrm{e}}$ depends on $\mu_{\mathrm{sp}}$ and $L_{\mathrm{m}}$.

Assuming quasi-static conditions, the reaction wrench of the environment can be found using the balance of forces:

$$
\left(W_{\mathrm{e}}\right)^{\mathrm{T}}=\left[-R_{\mathrm{b}}^{\mathrm{e}}\left[\begin{array}{c}
0 \\
0 \\
F_{\mathrm{u}}
\end{array}\right]+\left[\begin{array}{c}
0 \\
0 \\
m_{\mathrm{u}} g
\end{array}\right]\right],
$$

where $F_{\mathrm{u}}$ and $m_{\mathrm{u}}$ are the thrust and mass of the multirotor, respectively.

Since $I_{\mathrm{b}}$ is invertible, by combining and rearranging equations (1) and 2) the dynamics of the constrained system can be described as:

$$
\dot{T}_{\mathrm{b}}^{\mathrm{b}, \mathrm{e}}=f\left(T_{\mathrm{b}}^{\mathrm{b}, \mathrm{e}}, H_{\mathrm{b}}^{\mathrm{e}}, U, \mu_{\mathrm{sp}}\right),
$$

with $U:=\left[\tau_{\mathrm{x}}, \tau_{\mathrm{y}}, \tau_{\mathrm{z}}, F_{\mathrm{u}}\right]^{\mathrm{T}}$ being the input torques and thrust generated by the multirotor.

Due to the constraints imposed on the system, to stabilize the multirotor it suffices to stabilize its rotational dynamics, for which we consider a state-feedback controller. To apply such a controller, equation (3) is linearized around the equilibrium state given by:

$$
\begin{aligned}
\phi_{\mathrm{b}} & =0 ; \dot{\phi}_{\mathrm{b}}=0 ; \theta_{\mathrm{b}}=\theta_{\mathrm{sp}} ; \\
\dot{\theta}_{\mathrm{b}} & =0 ; \psi_{\mathrm{b}}=0 ; \dot{\psi}_{\mathrm{b}}=0 \\
\tau_{x, y, z} & =0 ; F_{\mathrm{u}}=F_{\mathrm{eq}} ; \mu_{\mathrm{c}}=\theta_{\mathrm{sp}} ;
\end{aligned}
$$

where the pitch setpoint $\theta_{\mathrm{sp}}$ is given as an input and $F_{\mathrm{eq}}$ is the thrust needed for the system to remain in equilibrium, given by:

$$
F_{\mathrm{eq}}=\frac{m_{\mathrm{u}} g}{\cos \left(\theta_{\mathrm{sp}}\right)}
$$

Note that, in the equilibrium configuration, the reader could estimate the normal force $F_{\mathrm{N}}$ applied by the system on the environment by using the following relation:

$$
F_{\mathrm{N}}=\frac{m_{\mathrm{u}} g}{\tan \left(\theta_{\mathrm{sp}}\right)} .
$$

We represent the linearized rotational dynamics as:

$$
\dot{X}=A\left(\theta_{\mathrm{sp}}\right)\left(X-X_{\mathrm{eq}}\right)+B\left(\theta_{\mathrm{sp}}\right)\left(U-U_{\mathrm{eq}}\right),
$$

with $X=\left[\begin{array}{llllll}\phi_{\mathrm{b}} & \dot{\phi}_{\mathrm{b}} & \theta_{\mathrm{b}} & \dot{\theta}_{\mathrm{b}} & \psi_{\mathrm{b}} & \dot{\psi}_{\mathrm{b}}\end{array}\right]^{\mathrm{T}}$ describing the angular state, and $X_{\text {eq }}$ and $U_{\text {eq }}$ are filled with the equilibrium values of equation (4).

The Linear Quadratic Regulator method, combined with gain-scheduling, can be applied to equation (7) to find stabilizing control gains $K\left(\theta_{\mathrm{sp}}\right)$ for each $\theta_{\mathrm{sp}}$ so that:

$$
U=K\left(\theta_{\mathrm{sp}}\right)\left(X_{\mathrm{sp}}-X\right)+U_{\mathrm{eq}}
$$

stabilizes the rotational dynamics of the system. This results in gain matrices of the following shape:

$$
K=\left[\begin{array}{cccccc}
K_{1,1} & K_{1,2} & 0 & 0 & K_{1,5} & K_{1,6} \\
0 & 0 & K_{2,3} & K_{2,4} & 0 & 0 \\
K_{3,1} & K_{3,2} & 0 & 0 & K_{3,5} & K_{3,6} \\
0 & 0 & K_{4,3} & K_{4,4} & 0 & 0
\end{array}\right],
$$

which indicate a distinct separation between the roll and yaw states, which are stabilized using the roll torque and yaw torque, and the pitch state which is stabilized by the pitch torque and the variation in thrust.

\section{EXPERIMENTS}

Experiments were performed to evaluate the multimodal approach towards surface cleaning (presented in Section III) and the control strategy (presented in Section IV), which are reported here. We have included a supplementary MP4 video showing the experiments, available at http://ieeexplore.ieee.org .

\section{A. Experimental setup}

The experimental setup consists of an aerial manipulator, ground-control station, Optitrack motion capture system and vertical surface. As shown in Figure 1, the aerial manipulator comprises a hexarotor platform, equipped with a single actuator manipulator which carries the end-effector presented in Section III. The system is controlled by an onboard Intel NUC i5 computer that communicates with the groundcontrol-station over a wireless network. The ground-control station provides the user interface to the operator. The motion capture system is used to obtain absolute pose measurements, which are used for the state estimation algorithm of the multirotor and to obtain experimental measurement results.

The hexarotor used in this work is illustrated in Figure 1. Its diameter (excluding propellers) is $80 \mathrm{~cm}$ and it weighs $2.1 \mathrm{~kg}$. A frame with relatively long arms was chosen deliberately to increase the gap between the front propellers to allow the manipulator to pass through. The hexarotor is controlled by a Pixhawk 2.1 Flight Controller, which runs the PX4 Firmware [17]. Its propulsion system consists of 30 A ESCs, Cobra CM2217 $950 \mathrm{Kv}$ motors and $10 \times 4.5$ inch dual-blade propellers. The aerial system is powered by a tethered $16 \mathrm{~V}$ power-supply. At this voltage the configuration can provide a maximum $F_{\mathrm{u}}$ of $78 \mathrm{~N}$.

The manipulator consists of a Dynamixel MX106R Servo Motor, which rotates along $\hat{y}_{\mathrm{b}}$, and a hollow carbon-fiber tube connected on top of this servo. The tube has a length of $60 \mathrm{~cm}$ and inner/outer diameters of $10.5-12 \mathrm{~mm}$, which provides sufficient structural stiffness to assume negligible deflection of the tube, given the weight of the end-effector. At the end of this rod a 3D-printed bend is attached that applies an angular correction as the rod is not exactly aligned with the vector $\vec{p}_{\text {be }}$. This bend connects to the elastic component of the end-effector. The manipulator has a weight of $0.23 \mathrm{~kg}$.

\section{B. Experiment description}

Two sets of experiments were conducted in a confined flying arena in which a wall was placed at $x_{\mathrm{w}}=1.6 \mathrm{~m}$ in the first and $x_{\mathrm{w}}=1.75 \mathrm{~m}$ in the second set. In both cases, the wall was aligned with the plane $\left(\hat{y}_{\mathrm{w}}, \hat{z_{\mathrm{w}}}\right)$ such that in interaction $\Psi_{w}$ and $\Psi_{e}$ have identical orientation.

In the first set of experiments the goal was to clean a scribble from the wall. This scribble was drawn on a $5 \times 10 \mathrm{~cm}(\mathrm{WxH})$ patch on the wall. An operator controlled 


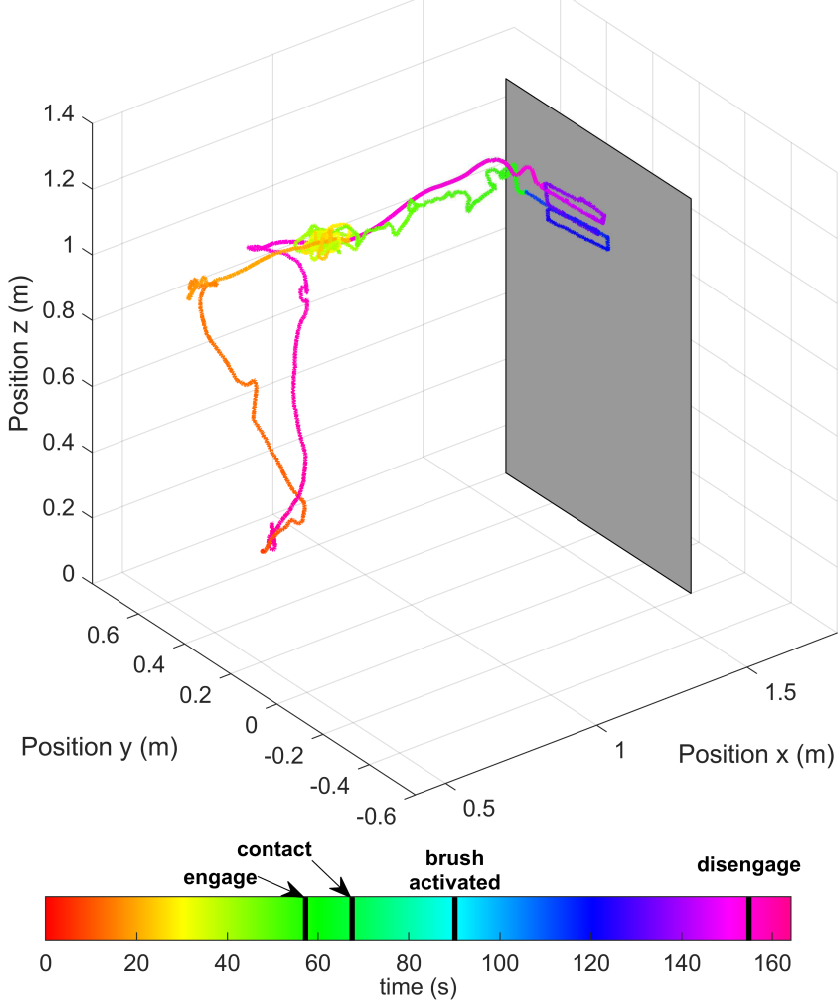

Fig. 6: The end-effector position over time in a surface cleaning experiment. Note that the end-effector is positioned on a single point from $t \approx 60 \mathrm{~s}$ to $t \approx 110 \mathrm{~s}$.

$p_{\mathrm{sp}}$ during the free-flight and engage phases, and $\theta_{\mathrm{sp}}$ during the engage and contact phases. To establish quick and reliable contact, $\theta_{\mathrm{sp}}$ was set to $20^{\circ}$ before entering the engage phase. Regarding the end-effector, the operator activated the brush and controlled the lateral movement by giving lateral velocity commands. The operator initiated the engage, contact and disengage phases in the experiment. Disengage distance $C$ was set to $0.75 \mathrm{~m}$.

In the second set of experiments, the repeatability of the approach was qualitatively evaluated. The switching control procedure was automated and repeated for several runs, each run lasting $64 \mathrm{~s}$. This procedure is as follows. First, the surface is approached by incremental adjustments of the $p_{\mathrm{sp}}$ in the free-flight phase. Then, the engage phase is initiated. Rather than using an immediate $20^{\circ}$ setpoint, $\theta_{\mathrm{sp}}$ is gradually increased from $12^{\circ}$ to $20^{\circ}$ over a period of 4 seconds to reduce the severity of impact. Then, the contact phase is initiated and $\theta_{\mathrm{sp}}$ is gradually increased further to $27^{\circ}$. An up-down locomotion is performed by sending a velocity command of 0.125 in both directions for $6 \mathrm{~s}$, with a pause of $2 \mathrm{~s}$ in between, after which the disengage is initiated.

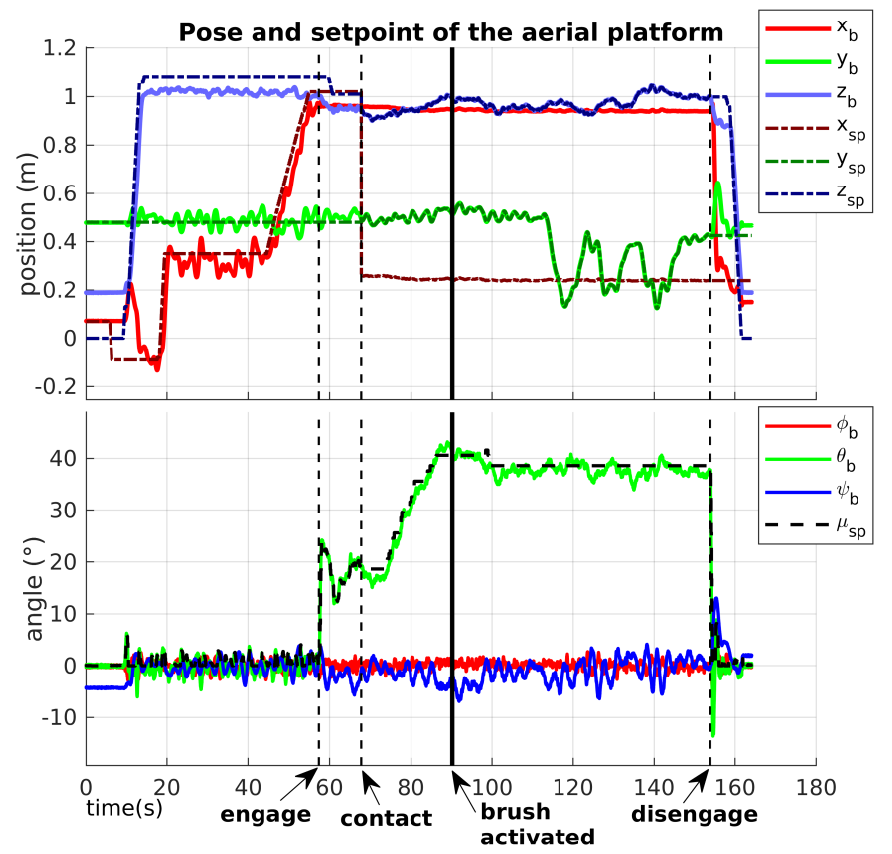

Fig. 7: The pose of the multirotor and the angle of the manipulator plotted over time in the surface cleaning experiment of Figure 6.
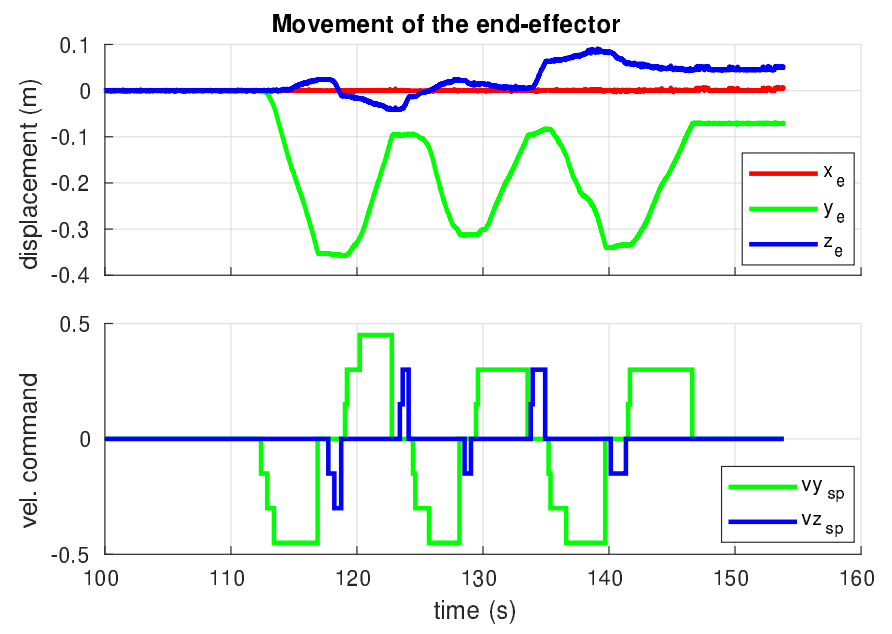

Fig. 8: The relative displacement of the end-effector and the associated velocity commands during the ground-locomotion phase in the experiment of Figure 6

\section{Results}

In the first set of experiments, several experiments were performed under contact angles varying between $25^{\circ}$ and $40^{\circ}$ to qualitatively evaluate the performance and reliability of the system in interaction. In all of the experiments the operator was able to clean the wall by controlling the ground locomotion, removing the scribbles.

Results of one experiment of the first set are displayed in Figures 6, 7 and 8 . Figure 6 visualizes the spatial position of the end-effector during the experiment; a part of the surface is illustrated for clarity. In Figure 7, the pose of the multirotor 
and the $\mu_{\mathrm{sp}}$ are plotted. Figure 8 shows the position and velocity commands of the end-effector. Different events are annotated the legend of Figure 6 and in the plots of Figure 7 .

The experiment starts by the system lifting off at $t=10 \mathrm{~s}$, ascending to approximately $1 \mathrm{~m}$. A small steady-state error between $z_{\mathrm{b}}$ and $z_{\mathrm{sp}}$ is visible due to a small estimation error of the system's mass and the lack of integral action in the altitude control loop. The multirotor is moved towards the surface and at $t=57.4 \mathrm{~s}$ the operator starts the engage and the system pitches forwards and successfully establishes contact. A small drop in height occurs before contact with the surface is made, which is caused by the end-effector mass not being taken into account in the contact controller. This drop causes a mismatch between $z_{\mathrm{sp}}$ and $z_{\mathrm{e}}$, which affects $\theta_{\mathrm{b}}$ and causes temporary contact-loss for the bottom wheel. In this experiment, the operator manually lowered $z_{\mathrm{sp}}$ to reduce this effect. After it stabilizes, the operator starts the contact phase at $t=67.8 \mathrm{~s}$. In contact, the controller solely depends on angular measurements and the position setpoints are reinitialized to accommodate the disengage phase. The operator gradually increases $\theta_{\mathrm{sp}}$ to the desired $40^{\circ}$ and activates the brush at $t \approx 90.7 \mathrm{~s}$. From $t=110 \mathrm{~s}$ to $t=150 \mathrm{~s}$ the operator actively controls the locomotion of the ground-system, moving it across the surface in the pattern as illustrated in Figure 6, effectively cleaning the drawing. In Figure 8, we observe that when pure horizontal velocity commands are given, also slight vertical displacements of the end-effector occur and vice-versa. These displacements are caused by occasional slipping of individual wheels and by undesired rotations of the end-effector on the surface. Although seemingly small, in several experiments the operator had to compensate for these displacements. After successfully cleaning the drawing, the operator initiates disengage at $t=154 \mathrm{~s}$, returns the system to free-flight and safely lands it. We observe that over the entire period in contact, $\phi_{\mathrm{b}}$ and $\psi_{\mathrm{b}}$ never exceed $7.5^{\circ}$.

One of the results of the second set of experiments is shown in Figure 9. In here, a total of 18 consecutive trials of the multimodal locomotion procedure were performed over a period of 20 minutes. The surface motion trajectories of each trial are shown in Figure 9a A high similarity between the motion profiles can be observed, with the exception of trials 7 and 16. However, we notice a sideways drift in the first point of attachment over the course of the experiment, for which we were unable to identify the cause. The controller effectively withstood disturbances and maintained stability in all 18 trials. In Figure $9 \mathrm{~b}$, the orientation of the multirotor and the pose of the end-effector are shown for trials 6, 7, 15 and 16. In trial 7 a coincidental bounce with the environment occurred during approach (see $x_{\mathrm{e}}$ ), which resulted in oscillations in $\theta_{\mathrm{b}}$, causing a vertical offset in the point of attachment (see $z_{\mathrm{e}}$ ). From the video is appears that the horizontal offset in the 16th trial is caused by a bounce due to disturbances as well, causing the system to make contact a bit further to the side. We notice no remarkable behavior in the plots for trial 16 .

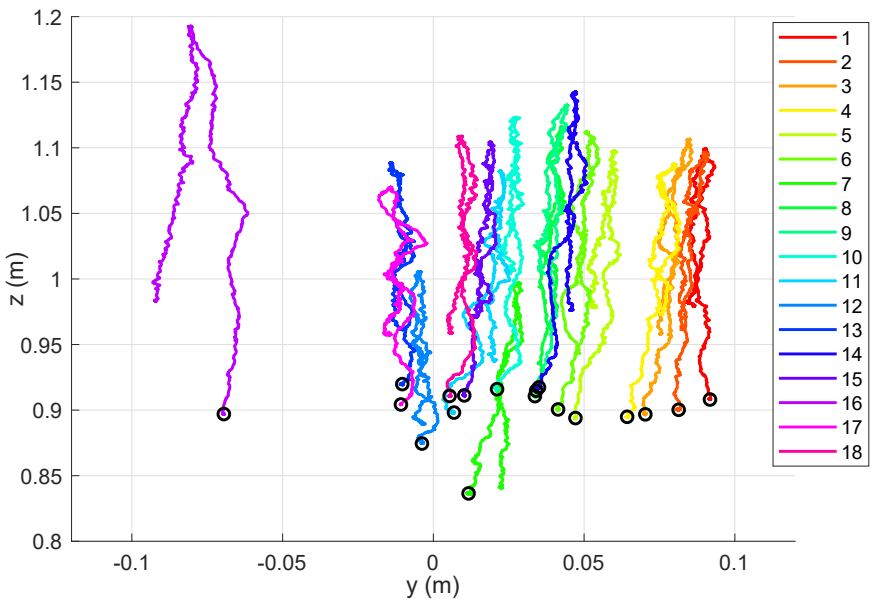

(a) The surface movement trajectories. A trend can be noted in the point of attachment over time, which is indicated by the circles.

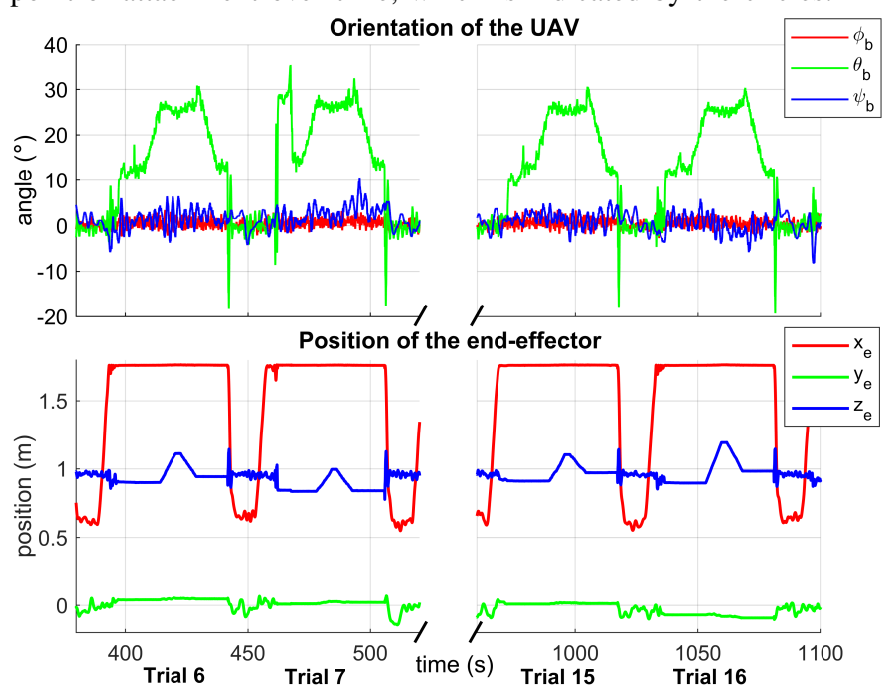

(b) The orientation of the multirotor and the position of the endeffector plotted for trials 6 and 7, and 15 and 16 .

Fig. 9: Results of the repeatability experiment in which 18 consecutive trials of the multimodal locomotion procedures are performed.

\section{Lessons learned}

Several lessons were learned from the extensive experimentation with the system, which are reported here such that these may be taken into account in future work:

- Slipping of wheels occurred frequently, resulting in uncontrolled rotations of the end-effector which caused additional disturbances to the multirotor. The groundlocomotion-system may benefit from a design with only two perpendicular actuated omni wheels, aligned such that slipping does not cause uncontrolled rotation. The rotation of the end-effector on the surface may be controlled solely by the multirotor and the elastic element.

- Under certain relative orientations between the endeffector and the multirotor, the elastic decoupling element applies an undesired torque. This can cause dis- 
connection of certain wheels, even if the net interaction force applied by the multirotor is properly aligned.

- The contact-controller demonstrates resilience to the disconnection of individual wheels from the surface, but becomes unstable whenever there is no contact with the environment for a brief moment. Therefore, persistence of contact is crucial, but persistent contact of all three wheels is not required.

- The contact-controller outputs desired torque and thrust values. Given the importance of aligning the interaction force to the approach, it is crucial to have an accurate mapping from rotor thrust to rotor velocities. This mapping depends on voltage, therefore the use of batteries (e.g. in outdoor scenarios) may pose additional challenges.

\section{CONCLUSION AND FUtURE WORK}

This paper proposes and evaluates a multimodal locomotion system as an approach to active tool handling on remote locations. The system has been designed as an aerial manipulator carrying an end-effector composed of three actuated omni wheels and a tool. The aerial manipulator represents the aerial-locomotion-system whereas the end-effector represents the ground-locomotion-system. The end-effector has been specifically designed to allow re-positioning of a tool on the surface. In order to deal with the issues related to the deployment this kind of platform, as described in the paper, a control strategy based on the authors' previous work has been modified and implemented on an experimental setup.

Experiments, in which a $5 \times 10 \mathrm{~cm}$ area was successfully cleaned with a $3 \mathrm{~cm}$ diameter brush, validate the approach. Results highlight that the disturbances on the aerial platform, introduced by the locomotion of the end-effector, are successfully counteracted by the applied control strategy, with the angular errors remaining below $7.5^{\circ}$. The repeatability of the multimodal locomotion approach is qualitatively demonstrated by an experiment in which 18 consecutive trials are stably performed.

A natural extension of this work is to extend the approach to surfaces of any orientation and curvature to allow application in a broader range of scenarios. Furthermore, strategies for trajectory generation can be investigated in order to seamlessly transition from free-flight into contact with the environment. Finally, the switching nature of the proposed control strategy should be studied.

\section{REFERENCES}

[1] F. Ruggiero, V. Lippiello, and A. Ollero, "Aerial manipulation: A literature review," IEEE Robotics and Automation Letters, vol. 3, no. 3, pp. 1957-1964, 2018.

[2] H. W. Wopereis, J. J. Hoekstra, T. H. Post, G. A. Folkertsma, S. Stramigioli, and M. Fumagalli, "Application of substantial and sustained force to vertical surfaces using a quadrotor," in Robotics and Automation (ICRA), 2017 IEEE International Conference on. IEEE, 2017, pp. 2704-2709.

[3] M. Fumagalli, R. Naldi, A. Macchelli, F. Forte, A. Q. Keemink, S. Stramigioli, R. Carloni, and L. Marconi, "Developing an aerial manipulator prototype: Physical interaction with the environment," IEEE robotics \& automation magazine, vol. 21, no. 3, pp. 41-50, 2014.
[4] M. Orsag, C. Korpela, S. Bogdan, and P. Oh, "Dexterous aerial robots - mobile manipulation using unmanned aerial systems," IEEE Transactions on Robotics, 2017.

[5] G. Gioioso, M. Ryll, D. Prattichizzo, H. H. Bülthoff, and A. Franchi, "Turning a near-hovering controlled quadrotor into a $3 \mathrm{~d}$ force effector," in Robotics and Automation (ICRA), 2014 IEEE International Conference on. IEEE, 2014, pp. 6278-6284.

[6] A. Albers, S. Trautmann, T. Howard, T. A. Nguyen, M. Frietsch, and C. Sauter, "Semi-autonomous flying robot for physical interaction with environment," in Robotics Automation and Mechatronics (RAM), 2010 IEEE Conference on. IEEE, 2010, pp. 441-446.

[7] C. Papachristos, K. Alexis, and A. Tzes, "Technical activities execution with a tiltrotor uas employing explicit model predictive control," IFAC Proceedings Volumes, vol. 47, no. 3, pp. $11036-11042,2014$.

[8] H. Tsukagoshi, M. Watanabe, T. Hamada, D. Ashlih, and R. Iizuka, "Aerial manipulator with perching and door-opening capability," in Robotics and Automation (ICRA), 2015 IEEE International Conference on. IEEE, 2015, pp. 4663-4668.

[9] M. Ryll, G. Muscio, F. Pierri, E. Cataldi, G. Antonelli, F. Caccavale, and A. Franchi, "6d physical interaction with a fully actuated aerial robot," in Robotics and Automation (ICRA), 2017 IEEE International Conference on. IEEE, 2017, pp. 5190-5195.

[10] S. Park, J. Her, J. Kim, and D. Lee, "Design, modeling and control of omni-directional aerial robot," in Intelligent Robots and Systems (IROS), 2016 IEEE/RSJ International Conference on. IEEE, 2016, pp. $1570-1575$

[11] H.-N. Nguyen, S. Park, and D. Lee, "Aerial tool operation system using quadrotors as rotating thrust generators," in Intelligent Robots and Systems (IROS), 2015 IEEE/RSJ International Conference on. IEEE 2015, pp. 1285-1291.

[12] J. L. Scholten, M. Fumagalli, S. Stramigioli, and R. Carloni, "Interaction control of an uav endowed with a manipulator," in Robotics and Automation (ICRA), 2013 IEEE International Conference on. IEEE, 2013, pp. 4910-4915.

[13] K. Alexis, G. Darivianakis, M. Burri, and R. Siegwart, "Aerial robotic contact-based inspection: planning and control," Autonomous Robots, vol. 40, no. 4, pp. 631-655, 2016.

[14] L. Daler, S. Mintchev, C. Stefanini, and D. Floreano, "A bioinspired multi-modal flying and walking robot," Bioinspiration \& biomimetics, vol. 10, no. 1, p. 016005, 2015.

[15] Y. Mulgaonkar, B. Araki, J.-s. Koh, L. Guerrero-Bonilla, D. M. Aukes, A. Makineni, M. T. Tolley, D. Rus, R. J. Wood, and V. Kumar, "The flying monkey: a mesoscale robot that can run, fly, and grasp," in Robotics and Automation (ICRA), 2016 IEEE International Conference on. IEEE, 2016, pp. 4672-4679.

[16] S. Stramigioli and H. Bruyninckx, "Geometry and screw theory for robotics," in Proceedings IEEE International Conference on Robotics and Automation. Citeseer, 2001.

[17] L. Meier, D. Honegger, and M. Pollefeys, "Px4: A node-based multithreaded open source robotics framework for deeply embedded platforms," in Robotics and Automation (ICRA), 2015 IEEE International Conference on. IEEE, 2015, pp. 6235-6240. 